\title{
Modeling of beam loss induced quenches in the LHC main dipole magnets
}

\author{
M. Breschi, Senior Member, IEEE, E. Felcini, F. Breccia, P.P. Granieri, E. Bergonzoni, A. Bevilacqua, P. Galassi, \\ T. Winkler, and L. Bottura
}

\begin{abstract}
The full energy exploitation of the Large Hadron Collider, a planned increase of the beam energy beyond the present 6.5 $\mathrm{TeV}$, will result in more demanding working conditions for the superconducting dipoles and quadrupoles operating in the machine. It is hence crucial to analyse, understand and predict the quench levels of these magnets for the required values of current and generated magnetic fields. A one-dimensional multi-strand electro-thermal model has been developed to analyse the effect of beam-losses heat deposition. Critical elements of the model are the ability to capture heat and current distribution among strands, and heat transfer to the superfluid helium bath.

The computational model has been benchmarked against experimental values of LHC quench limits measured at $6.5 \mathrm{TeV}$ for the MB (Main Bending) dipole magnets.
\end{abstract}

Index Terms-Beam losses, Accelerator Magnets, Quench, Superconductors, Magnet Stability, Rutherford cables

\section{INTRODUCTION}

$\mathbf{T}$ HE coils of the Main Bending (MB) magnets of the Large Hadron Collider (LHC) at CERN are wound with $\mathrm{Nb}-\mathrm{Ti}$ Rutherford cables [1], [2]. A relevant source of thermal disturbance in the operation of the accelerator magnets is the heat released on the superconducting wires by the phenomena deriving from the so called beam losses. When protons deviate from the nominal trajectory and interact with the inner surface of the vacuum chamber, they generate a shower of secondary particles. The interaction of these particles with the coils can lead to quenches, especially around the mid-plane [3]-[5]. The flux of secondary particles is measured by Beam Loss Monitors (BLMs) installed outside the magnet cryostats [5]. The measured data on these particle fluxes, and the consequent amount of energy released on the superconducting strands, must be compared with the cable stability margin, i.e. the minimum external energy density input that drives an irreversible transition to the normal state at given working conditions of the magnet. An accurate prediction of the stability margin is of primary importance for the definition of appropriate intervention thresholds of the BLM system in order to dump the particle beam [6].

Manuscript received and acceptance dates will be inserted here.

M. Breschi, is with the Laboratory of Magnet Technology and Applied Superconductivity of the University of Bologna, Bologna, 40136, Italy (e-mail: marco.breschi@unibo.it).

L. Bottura and E. Felcini are with the Technology Department, CERN, Geneva, Switzerland (luca.bottura@cern.ch, enrico.felcini@cern.ch).

F. Breccia, E. Bergonzoni, A. Bevilacqua, P. Galassi, were formerly with the University of Bologna, Italy (e-mail: francesca.breccia@gmail.com).
The complexity of this modeling is related to several aspects. First of all, the coupling of thermal, electromagnetic and hydraulic phenomena, involving current and heat redistribution between the strands, and the inherently non-linear behavior of the superconducting materials, make the analytical treatment of this problem impractical. Moreover, the peculiar feature of the heat deposit due to beam losses is that this is by nature nonuniform across the cable width [7], [8]. Modeling of the heat exchange with interstitial helium is particularly delicate since the porous LHC magnets are cooled with superfluid helium, which may undergo several phase transitions during a transient [9]-[13]. Furthermore, the heat exchange is of intrinsically transient nature, taking place during fast impulsive transients that significantly modify the coolant behavior with respect to steady state experiments [14]-[18]. Last but not least, the accurate modeling of this problem requires the knowledge of a significant number of parameters, such as the interstrand thermal and electrical conductances, the area of the cable cross section occupied by interstitial helium, the wetted perimeter of strands, the coefficients involved in the Kapitza heat transfer to superfluid helium, the level of heat flux leading to a boiling crisis, etc. In some cases, the values of these parameters reported in the literature are largely scattered or are included in rather broad ranges (see for example [19] for the interstrand electrical resistances in different types of cables).

The development of sophisticated numerical tools and a careful work to retrieve the model parameters are therefore essential to achieve the desired accuracy in the calculation of the stability margin. Significant achievements in these two directions have been accomplished in the last two decades [20]-[27].

This work describes a numerical model developed to tackle the computation of the stability margin of the $\mathrm{Nb}$ - $\mathrm{Ti}$ accelerator magnets of the LHC. The subsequent phases of quench propagation, detection and magnet dump are not investigated in the present study. This paper describes the model results, a discussion on the underlying physics, and the impact of the main numerical and physical parameters.

P.P. Granieri was formerly with CERN, and is now with Sécheron SA, Geneva, Switzerland and Mediterranean University of Reggio Calabria, Italy (email: pier.paolo.granieri@gmail.com).

T. Winkler is with the Energy, Materials and Systems Division, University of Twente, The Netherlands (t.winkler@utwente.nl). 


\section{From the 3-D Magnets to the 1-D Model}

The computation of the stability margin can be performed at different levels of approximation. The cables for superconducting accelerator magnets are by nature similar to multi-wire transmission lines, that are difficult to capture in a 2-D simulation. Therefore, an appropriate modeling of these conductors should be performed either in the frame of full 3-D model, or by means of a topological equivalent 1-D model. In this work we follow the latter approach, based on the assumption introduced in [28], [29] that the components in the cable have a large ratio of length to cross-sectional dimensions. With this approach, the cable strands and the interstitial helium are modelled as parallel components and the model equations can be written in 1-D. The topological structure of the thermal and electric 1-D model is equivalent to that of the 3-D cable. The 1D model therefore reflects the geometric configuration and the pattern of contacts between electric and thermal elements of the real conductor.

The model adopted here is based on the general electromagnetic, thermal and hydraulic model described in [29], and implemented in the THEA code [30]. The specific model features adopted for the computation of the stability margin, the determination of the model parameters and the simulations setup are described in the next sections.

\section{A. Electro-thermal stability model}

For stability modelling, the system is split into three main physical domains: electromagnetic, thermal and hydraulic, each composed of a set of interacting elements. The electromagnetic domain includes $N$ elements, corresponding to the $N$ composite wires of the superconducting cable (each made of $\mathrm{Nb}$-Ti filaments and copper/bronze stabilizer). The thermal domain includes $N+1$ elements, corresponding to the $N_{s}$ composite strands plus the interstitial helium. The hydraulic model is composed of only 1 element, corresponding to the cooling bath.

In the electromagnetic model, each superconductive wire is described assuming the $\mathrm{Nb}$-Ti filaments and the metallic stabilizer in parallel electrical connection, and using a power law for the superconductor $E$ - $J$ characteristics. The cable is described through a distributed parameters electric circuit, in which the strands are connected to each other through contact conductances and mutual inductances, taken uniform along the cable length. The electromagnetic model, applied in [31] to compute the long-range coupling currents in Rutherford cables, was recently modified to allow the computation of short-range coupling currents and corresponding losses [32].

In the thermal model, the strands are cross linked via contact conductances, taken uniform along the cable length. The strands are also thermally linked to the helium bath and the interstitial helium. By helium bath we refer here to the annular channel of superfluid helium located between the outer surface of the vacuum chamber and the inner surface of the coils, as shown in Fig. 1. We indicate the part of superfluid helium which, percolating from the bath through the electric insulation, gets in direct contact with each strand of the porous Rutherford cable as interstitial helium.

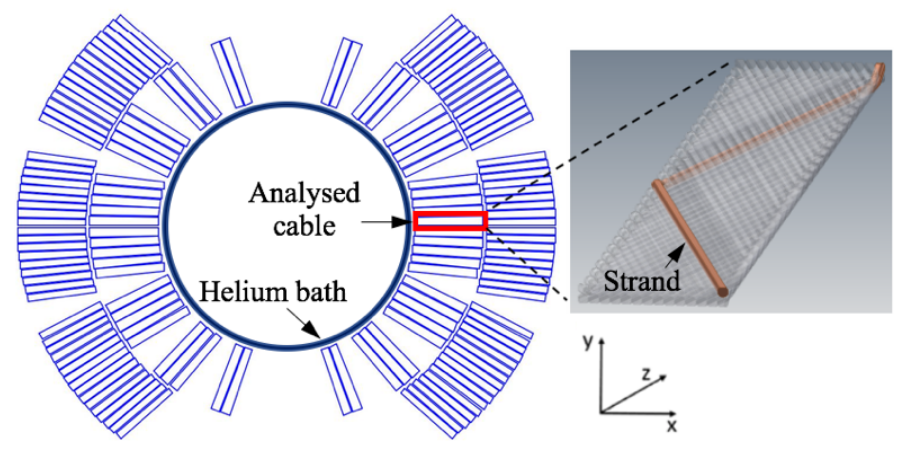

Fig. 1. LHC Main Dipole cross section, with a sketch of the path of one strand of the Rutherford cable along the $z$ direction.

The models of the heat exchange with the helium bath and the interstitial helium are described in the next section. Finally, in the hydraulic model, the helium bath is considered as a reservoir at a constant temperature of $1.9 \mathrm{~K}$.

\section{B. Heat exchange with liquid helium}

The heat transfer through the cable electrical insulation to the helium bath occurs at the small side of the cable. This mechanism is described here by means of a steady state heat transfer coefficient, since no experimental data are available for this geometry in transient conditions for very high pulsed heat depositions. Given the high characteristic time scale of thermal exchange between strands and helium bath, no relevant contribution to the magnet stability are expected in transient conditions. The heat transfer coefficient is based on experimental results on stacks of Rutherford cables subjected to a radially and longitudinally uniform heat deposit [13], scaled to the coil geometry [33].

The heat exchange between strands and interstitial helium is based on a transient heat transfer model, which subdivides the transition from $1.9 \mathrm{~K}$ to gaseous state into three main phases with different heat transfer coefficients.

The first phase, described by the Kapitza heat transfer coefficient, expressed as in [34], is included in the temperature range from $1.9 \mathrm{~K}$ to $T_{\lambda}$, the phase transition temperature from $\mathrm{He}$ II to He I. When the helium temperature is below $T_{\lambda}$, but the cable temperature is significantly higher, the heat flux may reach extremely high values, which can cause a boiling crisis at the interface between helium and strands. In the reference model, the boiling crisis criterion is set when a limiting flux of $100 \mathrm{~kW} / \mathrm{m}^{2}$ is reached (see [18], [35], [36]). Some variants of this threshold criterion for the boiling crisis are discussed in Section IV. The second phase is included in the temperature range from $T_{\lambda}$ to the He I saturation temperature, and is described by the heat transfer coefficient to liquid helium I reported in [36]. As a third phase, for helium temperature above the He I saturation temperature, a heat transfer coefficient to helium gas is modelled, with a constant value set to $70 \mathrm{~W} / \mathrm{m}^{2} \mathrm{~K}$ [34]. An initial analysis on the impact of the nucleate and film boiling phases showed that these do not significantly affect the stability margin. Thus, these two regimes have been neglected in the model adopted here. 


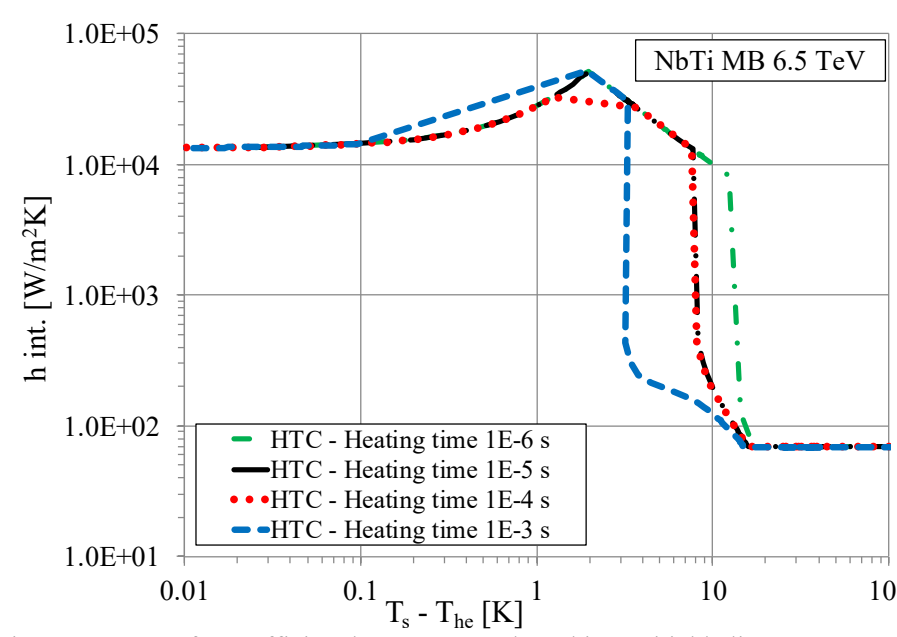

Fig. 2. Heat transfer coefficient between strands and interstitial helium vs temperature difference during different quench simulations for the MB magnet mid-plane cable operating at $6.5 \mathrm{TeV}$.

The heat transfer coefficient towards interstitial helium $h_{\text {int }}$ is plotted in Fig. 2 as a function of the difference between the temperature of the strands $T_{s}$ and the interstitial helium temperature $T_{h e}$. The first drop of $h_{\text {int }}$ corresponds to reaching the critical heat flux in the Kapitza first phase of heat exchange. The second drop corresponds to the helium phase transition at $T_{\lambda}$, with the beginning of the second phase.

The evolution of the boundary layer is time dependent, hence different profiles of $h_{\text {int }}$ can be observed in He I regime, depending on the considered time scale. For large temperature differences, the third phase with a very low heat exchange coefficient towards the helium bath is established.

\section{Simulations setup and main parameters}

The beam losses represent distributed heat depositions, taking place over significantly long sections of the magnets, in the order of several meters or tens of meters [5].

In the simulations the heat is deposited on the composite strands along the central 1 meter of a 2 meter long cable. The boundary conditions of the thermal model are set by imposing a constant temperature of $1.9 \mathrm{~K}$ at one cable end and an adiabatic condition at the other end (see Fig. 3). Thanks to the symmetry condition, this choice is equivalent to deposit heat over a $2 \mathrm{~m}$ long region around the center of a $4 \mathrm{~m}$ long cable. A set of simulations showed that using longer cable lengths leads to the same results, while requiring larger computation resources.

As for the electromagnetic model, a uniform current distribution is imposed at both cable ends. The profile in time of the heat deposition is a rectangular pulse in the range from $1 \mu$ s to $10 \mathrm{~s}$, denoted here as heating time.

Both the magnetic field and the heat deposition exhibit a 2-D non-uniform distribution in the magnet cross section. The model accounts for these distributions, which are assumed identical at every cross section along the magnet length.

The simulations described hereafter are focused on the cable located in the inner layer at the mid-plane of the magnet (see Fig. 1), as this is subjected to the highest heat deposit [5], [37].

The magnetic field on the cable cross section decreases linearly across the cable width in the radial direction.
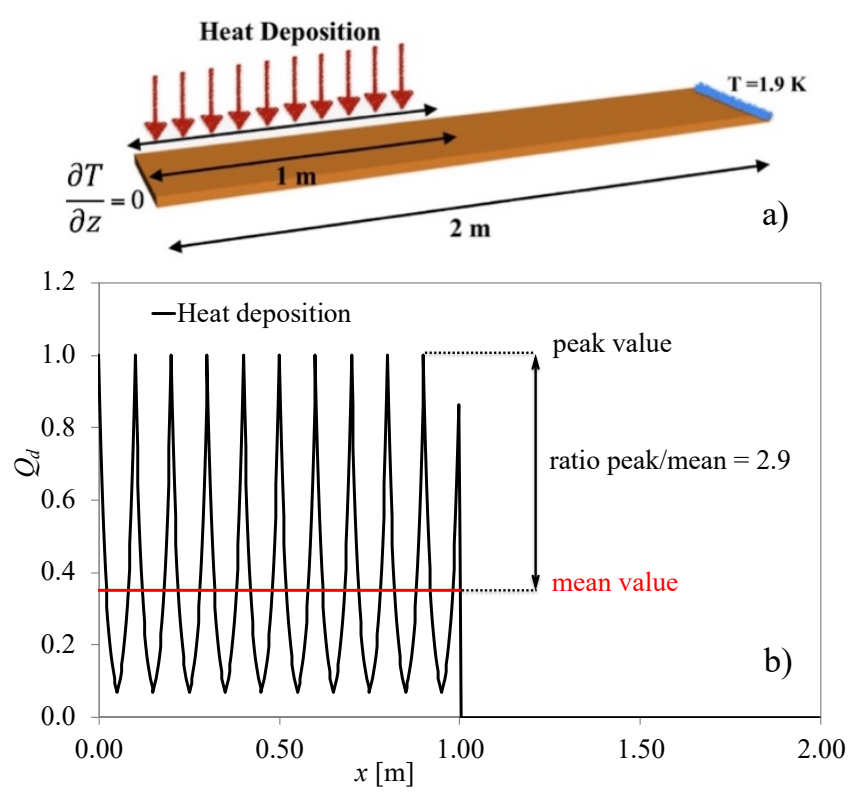

Fig. 3. a) Region of heat deposition along the cable length and boundary conditions of the thermal model. b) Adimensional profile of the heat deposition along the axis of one strand.

The heat deposition exhibits a nearly exponential decay across the cable width, as described in [38].

Since the strands are transposed with a given pitch $L_{p}$, as shown in Fig.1, each of them is subjected to variable field and heat depositions along its length, characterized by a periodic oscillation with a period equal to $L_{p}$. From the magnetic field distribution in the magnet cross section, the magnetic field along a given strand is computed. Similarly, from the maps of heat deposit on the magnet cross-section [5], the longitudinal profile of the disturbance along each wire can be computed. The adimensional profile of the heat deposition is reported in Fig. $3 \mathrm{~b}$. The ratio between the peak and the mean value of the heat deposition distribution is about 2.9. In the simulations with a non-uniform heat deposition, this adimensional distribution is multiplied by a given value of per unit length power. In order to compute the quench energy, an iterative procedure is adopted. The initial value of per unit length power is increased if the result of the simulation is a recovery, and reduced in case of quench, until the difference between the maximum recovery energy and the minimum quench energy is below $5 \%$.

The area available for the interstitial helium is computed from the geometry of the Rutherford cable cross section and derived from indirect experimental investigations [40]. The wetted perimeter is also derived from geometric considerations and by rescaling the results obtained in [27] with neutron tomography.

In a simplified version, the 1-D model takes only one strand as representative of the whole conductor behaviour [38]. In this case, a fraction $1 / N$ of the available interstitial helium is considered in the simulations. In the 1-strand model, the only thermal elements involved are the strand and the interstitial helium. The strand can transfer heat to the interstitial helium and the helium bath, but cannot exchange current and heat with the other wires. The magnetic field and heat deposition profiles are the same as those adopted for the $N$-strand model. 


\section{RESUlTS AND DisCUSSION}

The model was benchmarked against experimental values of the LHC quench limits reconstructed from the machine operation quenches at $6.5 \mathrm{TeV}$ of beam energy for the MB (Main Bending) dipole magnets [38]. The model was able to reproduce the experimental results over a wide range of heat deposition times (as shown in Section IV.B), and was therefore applied to compare different beam energies and to the analysis of the LHC Main Quadrupole [41].

In this Section, we apply the validated model to analyze the physical mechanisms occurring during the initial phases of quench at different operating conditions of the LHC MB dipoles. The simulated quench energy density, or simply quench energy, expressed in $\mathrm{mJ}$ per cubic centimeters $[\mathrm{mJ} / \mathrm{cc}]$, is presented in Fig. 4 as a function of the heating time. We compare the results obtained for the MB magnet at the transport current corresponding to a beam energy of $6.5 \mathrm{TeV}\left(I_{o p}=11 \mathrm{kA}\right)$ with those computed at a low operating current level, corresponding to the beam injection in the LHC $\left(I_{o p}=761 \mathrm{~A}\right)$.

The stability margins in the figure are computed as the peak power of the heat deposition in the mid-plane cable inner radial position, multiplied by the heat deposition time, and divided by the total cable volume. The same computations were also performed by applying a uniform heat deposition along each strand, i.e. a constant heat deposition profile across the cable width. The computed stability margins are compared with the sum of the enthalpies of the strands (indicated as strands enthalpy) and with the sum of the enthalpies of the strands and the interstitial helium (indicated as cable enthalpy). The enthalpy reserve is computed between the initial temperature of $1.9 \mathrm{~K}$ and the current sharing temperature corresponding to the peak field applied to the strands.

At nominal operation current and fast pulses, the stability margin overcomes the enthalpy of the strands, due to the heat exchange with interstitial helium in the Kapitza regime. This holds true both considering the non-uniform and the uniform heat deposition computations. In these conditions the ratio between the results obtained with non-uniform heat deposition and with uniform heat deposition is much less than 2.9.

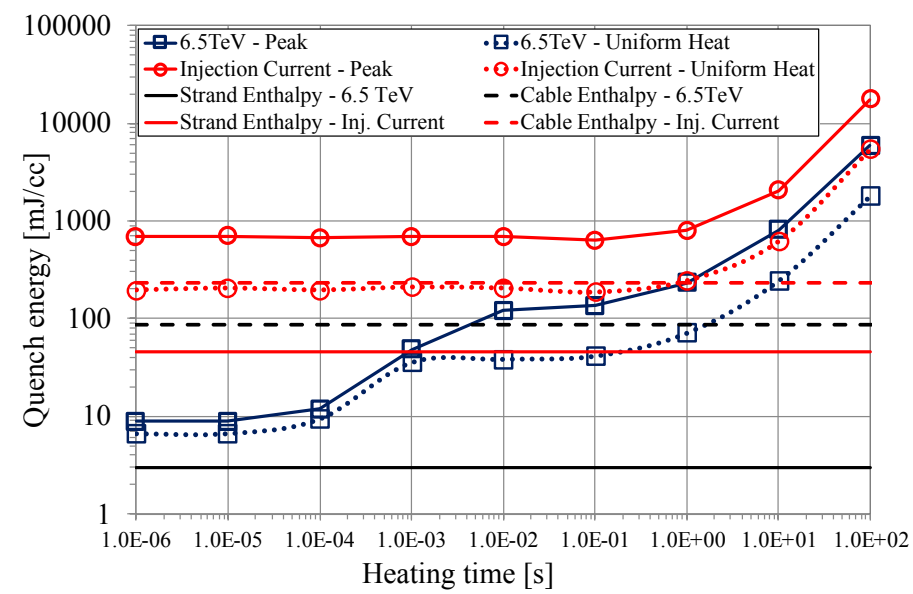

Fig. 4. Values of the stability margin of a LHC MB dipole inner layer cable at nominal current and nominal field $(6.5 \mathrm{TeV})$ and injection current $\left(I_{o p}=761 \mathrm{~A}\right)$ in the magnet mid-plane.
For these heating times, the local heat deposition peaks are driving the magnet stability.For pulse durations in the range from $1 \mathrm{~ms}$ to $0.1 \mathrm{~s}$, and a uniform heat deposition, the boiling crisis impedes the full exploitation of the available enthalpy up to the current sharing temperature $T_{c s}$, which is therefore only reached with longer heat deposition times.

At injection current, for heat deposition times below $0.1 \mathrm{~s}$, the stability margin computed with a uniform heat deposition practically corresponds to the value of the cable enthalpy.

For heat pulses longer than $0.1 \mathrm{~s}$, the cooling from the helium bath starts playing a role, and the stability margin significantly overcomes the cable enthalpy. At low current, the results of the peak curve lie above those of the uniform deposition case. The ratio between the two curves is almost constant along the heating time scale and equal to 2.9 , which corresponds to the ratio between the peak value of the non-uniform heat deposition and its mean value, as shown in Fig. 3b. In other words, at low current the stability is driven by the total amount of deposited energy, regardless of its distribution.

Further information can be derived from the analysis of the temperature curves. Fig. 5a reports, for the single-strand model, the strand and interstitial helium temperatures at the hotspot (where the adiabatic condition is applied) for the two extreme cases of highest energy input leading to recovery and lowest energy input leading to quench at the nominal operation current of the MB inner layer cable, with a $1 \mu$ s heat pulse. The computed strand temperatures in these two cases practically overlap until a given quench decision time, after which the temperatures decrease in case of recovery and run away in case of quench.

For nominal transport currents in the MB dipole mid-plane inner layer cable, the quench decision time is around $100 \mu \mathrm{s}$, which is greater than the heat deposition time. The cooling by the interstitial helium is not limited to the very short time of the heat pulse, but can act over longer times and thus contribute to increase the stability margin with respect to the strands enthalpy. At the quench decision time at high transport currents, the helium is still relatively cold (generally below $2.16 \mathrm{~K}$ ).

The thermal runaway occurs due to the fact that the temperature difference between the helium and the strands gets so high that a boiling crisis takes place.

At the low operation current level corresponding to injection $\left(I_{o p}=761 \mathrm{~A}\right)$, the Joule power to be extracted from the cable is much lower, and even the helium in gaseous state can effectively extract it, notwithstanding the low heat transfer coefficient. As shown in Fig. 5b for a $100 \mu$ s heat pulse, the quench decision time in these working conditions is generally higher, around 0.1 , and the bifurcation between the quench and recovery curves of the strand occurs for helium temperatures significantly greater than in the case of high transport currents. This separation generally occurs when the helium temperature approaches the current sharing temperature. At that point, the temperature difference between the interstitial helium and the cable strands becomes too low for an efficient heat extraction, and the thermal runaway takes place.

This phenomenon sets the quench energy very close to the cable enthalpy, as already observed. 

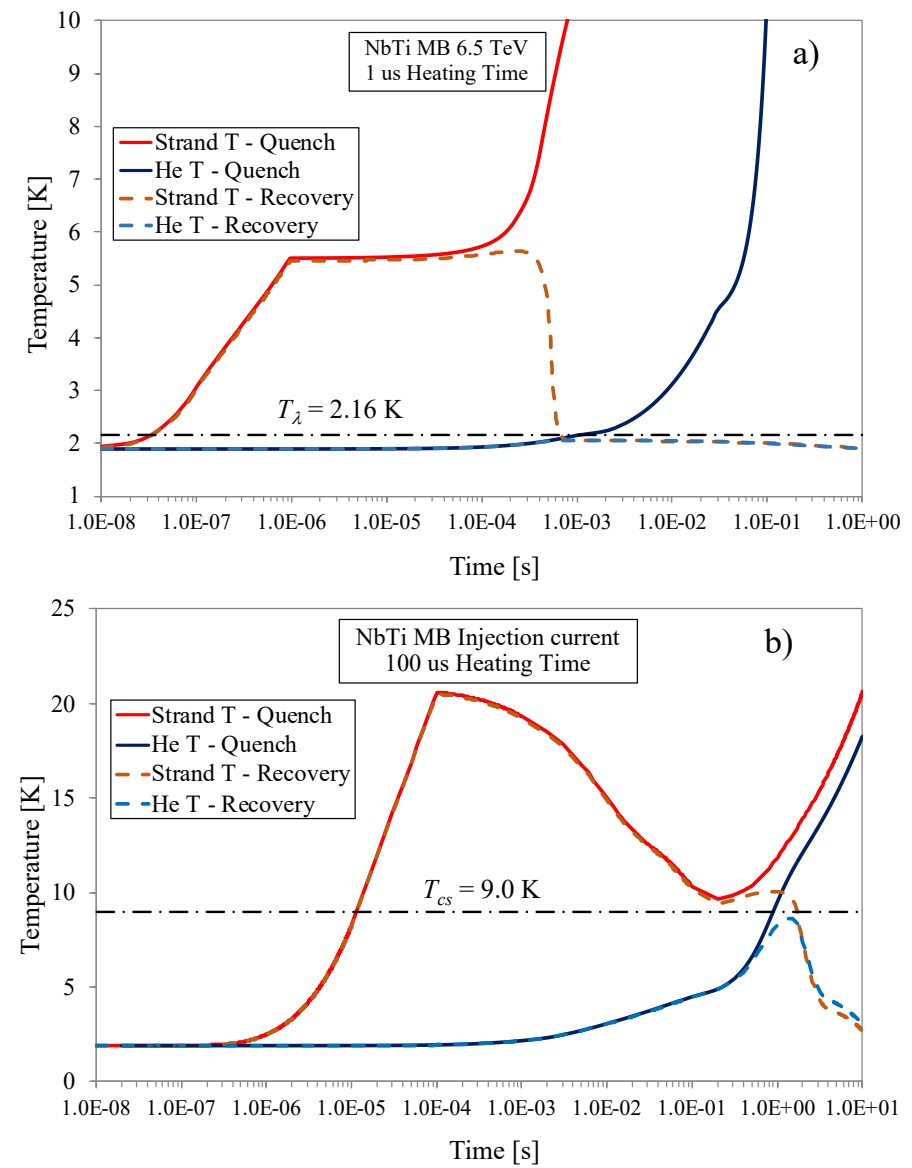

Fig. 5. Time dependence of the strand temperature and interstitial helium temperature at the hotspot in the stability simulation of a LHC MB inner layer cable for a) a $1 \mu \mathrm{s}$ heat pulse at nominal current $\left(I_{o p}=11 \mathrm{kA}\right)$ and b) a $100 \mu \mathrm{s}$ heat pulse at injection current $\left(I_{o p}=761 \mathrm{~A}\right)$. The pulse starts at $t=0 \mathrm{~s}$.

\section{PARAMETRIC StUdies}

\section{A. Numerical parameters}

To ensure the consistence of the numerical results, a convergence study was carried out to identify the impact of the numerical parameters on the simulation results, namely minimum and maximum integration time steps and mesh size. The simulations were performed with a Euler Backward time integration method, uniform mesh of 400 nodes including linear mesh elements, and with an adaptive time step between a minimum and a maximum value. The convergence study was performed both with and without electrical connection between the strands. Since the results obtained are similar, it can be stated that, due to the shorter time constants of thermal diffusion, in the range from $\mu$ s to ms, (see [28]), this is the dominant phenomenon in determining the conditions for numerical convergence.

Analysing the dependence of the stability margin on the maximum integration time step, it was found that the convergence is reached for all simulations with a maximum step of $10^{-7} \mathrm{~s}$. As shown in Fig. 6, the request on the maximum integration time step can significantly be relaxed at higher values of the heating time. For heat depositions longer than $1 \mathrm{~ms}$, maximum time steps in the order of $10^{-6} \mathrm{~s}$ are sufficient to reach convergence. As for the minimum time step, the convergence is reached for
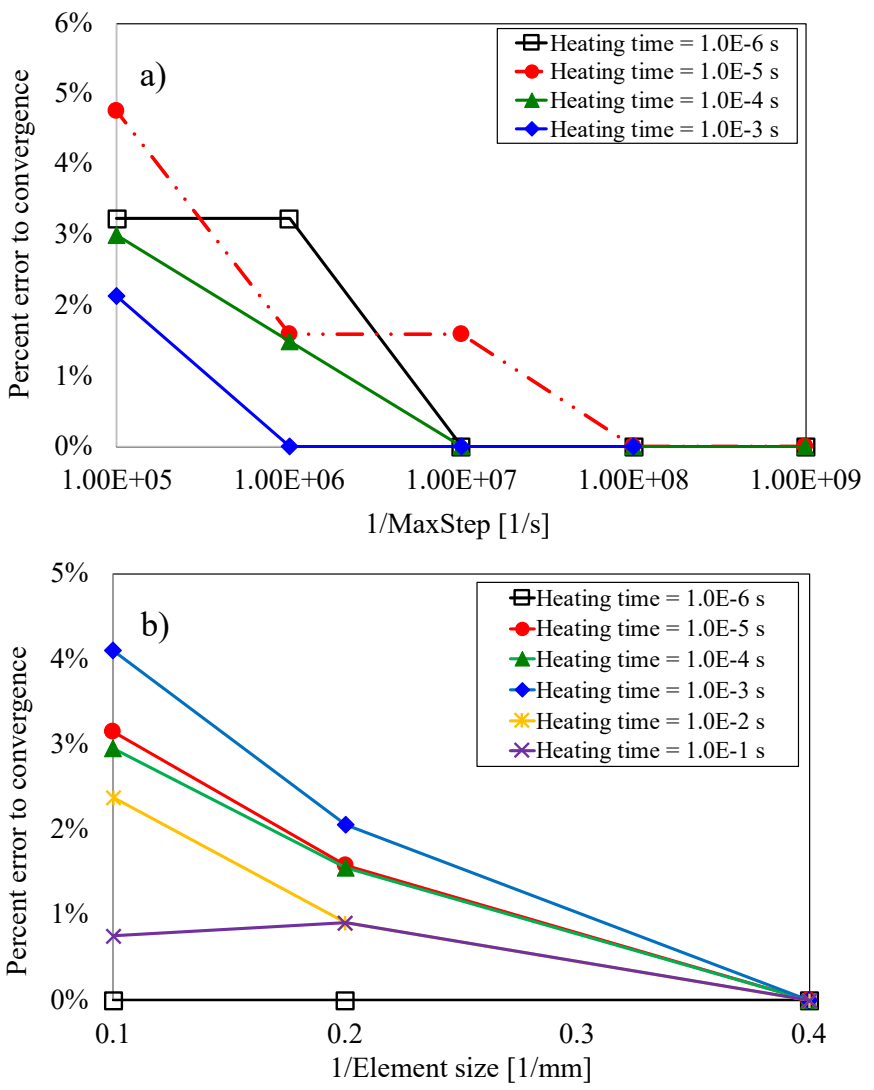

Fig. 6. Impact on the error with respect to convergence of the a) maximum time step and b) mesh element size set for the numerical integration.

the shortest heat pulses of $1 \mu \mathrm{s}$ with minimum steps below $10^{-8}$ s. This condition can be relaxed for longer heat deposition times.

The simulations presented here were therefore performed with variable minimum and maximum time step, subdividing each run at given working conditions in different phases in time, to reduce the computational effort without affecting the results [42].

A convergence study concerning the mesh element size, reported in Fig. 6b, showed that the numerical convergence is reached for mesh elements smaller than $5 \mathrm{~mm}$, which were therefore selected for all simulations presented here.

\section{B. Physical parameters}

As already mentioned in the introduction, the model results are strongly affected by the values of the main physical parameters. A set of simulations were run to assess the impact of these physical parameters on the model results.

The most crucial parameters affecting the stability margin at short pulse durations are those related to the heat exchange with interstitial helium, such as the wetted perimeter, the interstitial helium area, and the criterion selected for the onset of the boiling crisis. For longer heat deposition times, the pivotal parameters are those intervening in the description of the heat exchange with the helium bath.

In order to quantify the impact of the heat exchange between the strands and the interstitial helium, different variants of the model were adopted, and the results are presented in Fig. 7. 


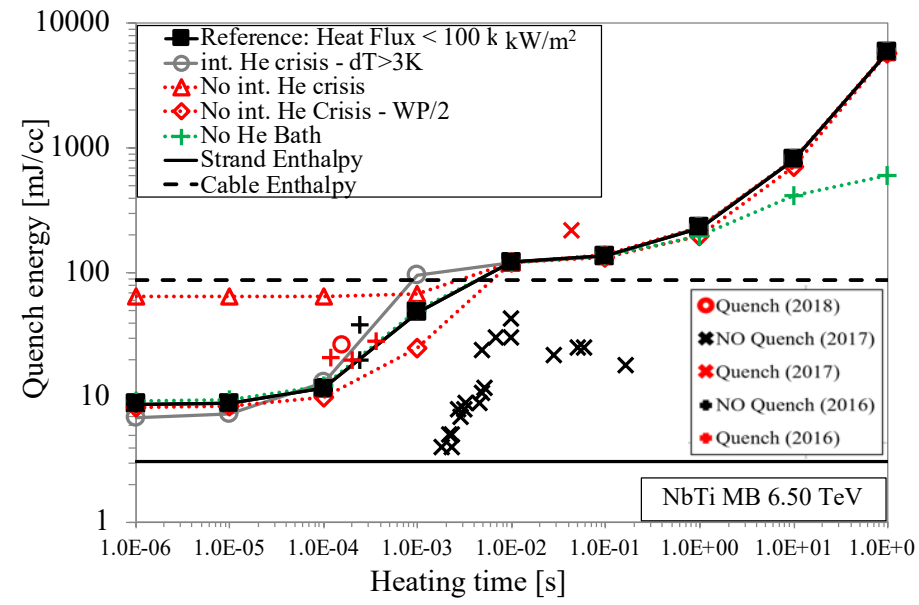

Fig. 7. Impact of the heat exchange between strands and interstitial helium and helium bath on the stability margin of the MB LHC dipole at $6.5 \mathrm{TeV}$.

The reference model (shown also in Fig. 4 as $6.5 \mathrm{TeV}$-Peak) considers the boiling crisis onset, at helium temperatures below $T_{\lambda}$, for heat fluxes exceeding $100 \mathrm{~kW} / \mathrm{m}^{2}$. Given the scattering of the boiling crisis onset criteria found in the literature, a different model was defined by imposing an upper limit to the temperature difference between the strands and the helium. This limit was set to $3 \mathrm{~K}$, according to [35].

The black and the grey lines in Fig. 7 define the margin of uncertainty on the mechanism of heat exchange between strands and interstitial helium. It is possible that other combinations of the model parameters result in even greater margins of uncertainty. Fig. 7 also reports quench and no-quench energy data points reconstructed from the LHC machine operation in the three years from 2016 to 2018, as explained in detail in [41].

Despite the uncertainties, the model is able to predict with a good accuracy the MB magnets quench limits.

Still, it is important to analyse the impact of the main physical parameters affecting the stability margin of the cable. The absence of a boiling crisis criterion in the model at helium temperatures below $T_{\lambda}$ can lead to unphysical heat exchange, resulting in an overestimation of the stability margin by one order of magnitude for fast pulses, as indicated by the dotted red line with triangles.

Furthermore, the wetted perimeter (WP) between strands and interstitial helium is assumed uniform across the cable width and, for the reference model, it is the average value of the distribution measured in [27]. For highly compressed cables, the wetted perimeter could be further reduced to lower values. In addition, it is very difficult to assess a reference value for all the Main Dipoles in the LHC, since quenches, cool down and powering can modify the internal geometry of these porous cables.

To evaluate the effect of possible wetted perimeter reductions, the simplified model without helium crisis has been simulated with half of the reference WP and Fig. 7 shows that the stability margin suffers a severe decrease (dotted red line with squares). We can state that the heat exchange between strands and interstitial helium strongly affects the stability margin for heating times up to about $10 \mathrm{~ms}$.
For longer deposition times the details of the heat exchange with interstitial helium do not affect the results, and the curves obtained with the various models overlap.

In order to evaluate the impact of the heat exchange with the helium bath, the reference model was adopted to describe the heat transfer to the interstitial helium, but no heat exchange with the helium bath was assumed. Comparing the results obtained with and without helium bath in Fig. 7, we can conclude that the helium bath starts improving the cable stability only for deposition times greater than $0.1 \mathrm{~s}$.

\section{CONCLUSION}

The computation of the stability margin of the LHC magnets is a complex task which requires sophisticated analysis tools and a great care in the selection of the model parameters. A model, and its corresponding parameters, have been identified that reproduce satisfactorily the quench energy values reconstructed for the MB magnets from the operation data of the LHC machine.

Three main aspects have been found crucial for the model effectiveness. The description of the heat exchange with the interstitial helium is of great importance for the determination of the stability margin with respect to fast pulses. In particular, the heat exchange coefficient in the Kapitza regime must be limited below a critical value leading to the boiling crisis. A second relevant aspect is to account for the non-uniformities of the magnetic field and of the heat deposition in the magnet cross section, which translate into non-uniformities along the length of each cable strand. Finally, the modelling of the current and heat redistribution between strands plays a significant role for intermediate pulse durations and high transport currents in the LHC MB magnet.

The model allows a better qualitative and quantitative understanding of the phenomena occurring during the quench in the LHC MB magnets. The quench at high current levels occurs mainly due to the boiling crisis, with the helium still at temperatures below the lambda transition temperature and quench decision times of the order of hundreds of microseconds. At low current levels, the quench occurs at much higher helium temperatures, corresponding to the current sharing temperature of the superconductor (about $9 \mathrm{~K}$ ). The quench decision time in these cases is much greater than for high currents, and is in the order of seconds.

This detailed model allows one to identify working conditions, such as the low current level corresponding to injection, or the very long heat deposition times, at which the details of the heat deposition profile have no impact, so that simpler models can be adequate for a fast and accurate calculation of the magnet stability margins.

\section{ACKNOWLEDGMENT}

A. Lechner, D. Richter, G. Willering, C. Scheuerlein from CERN are gratefully acknowledged for useful discussions. 


\section{REFERENCES}

[1] L. Rossi, "The LHC superconducting magnets", Proceedings of the Particle Accelerator Conference, PAC 2003, 2003.

[2] L. Rossi, "Superconductivity: its role, its success and its setbacks in the Large Hadron Collider of CERN", Supercond. Sci. Technol., vol. 23, 034001, 2010.

[3] G. Papotti et al., Macroparticle-induced losses during 6.5 TeV LHC operation, in Proceedings of the 7th Int. Particle Accelerator Conf. (IPAC18), Busan, South Korea, May 2016, pp. 1481-1484.

[4] A. Lechner et al., BLM Thresholds and UFOs, in Proceedings of the 7th Evian Workshop on LHC Beam Operation, Evian Les Bains, France, 2016, pp. 209-214.

[5] A. Priebe, "Quench tests of LHC magnets with beam: studies on beam loss development and determination of quench levels," PhD Thesis, Ecole Polytechnique Federale de Lausanne, Lausanne, Switzerland, 2014.

[6] B. Auchmann et al., "Testing beam-induced quench levels of LHC superconducting magnets", Phys. Rev. ST Accel. Beams, vol. 18, 061002.

[7] J. B. Jeanneret, D. Leroy, L. Oberli, T. Trenkler, "Quench levels and transient beam losses in LHC magnets", LHC Project Report 44

[8] C. Kurfurst, "Quench protection of the LHC quadrupole magnets," Master thesis, Vienna University of Technology, 2010

[9] S. W. Van Sciver, Helium cryogenics, The international cryogenics monograph series, New York, USA, 1986.

[10] J. G. Weisend, Handbook of cryogenic engineering, Taylor and Francis, Philadelphia, USA, 1998.

[11] T. M. Flynn, Cryogenic engineering, Taylor and Francis, Boca Raton, Florida, USA, 2005.

[12] N. S. Snider, "Heat transport through helium II: kapitza conductance," Cryogenics, vol. 10 (2), pp. 89-95, 1970.

[13] P. P. Granieri, "Heat Transfer between the Superconducting Cables of the LHC Accelerator Magnets and the Superfluid Helium Bath," PhD. Thesis, Ecole Polytechnique Federale de Lausanne, Switzerland, 2012.

[14] W. G. Steward, "Transient helium heat transfer phase I - static coolant," International Journal of Heat and Mass Transfer, vol. 21, pp. 863 - 874, 1978.

[15] O. Tsukamoto, S. Kobayashi, "Transient heat transfer characteristics of liquid helium," Jour. Appl. Physics, 46, 1975.

[16] C. Schmidt, "Transient heat transfer to liquid helium and temperature measurement with a response time in the microsecond region," Applied Physics Letters, vol. 32 (12), pp. 828 - 829, 1978.

[17] V. I. Deev, et al., "Transient boiling crisis of cryogenic liquids," International Journal of Heat and Mass Transfer, vol. 47, pp. 5477 - 5482, 2004.

[18] B.A. Danilchenko, M.O. Lutset and V.N. Poroshin, "Limit of transient heat absorption by superfluid helium for very large heat pulses," Cryogenics, Vol. 29, 1989.

[19] G. P. Willering, A. P. Verweij, J. Kaugerts, H. H. J. ten Kate, "Stability of Nb-Ti Rutherford Cables Exhibiting Different Contact Resistances," IEEE Trans. Appl. Supercond., vol. 18, no. 2, pp. 1263 - 1266, 2008.

[20] D. E. Baynham, D. A. Cragg, D. C. Coombs, P. Bauer, and R. Wolf, "Transient stability of LHC strands," IEEE Trans. Appl. Supercond., vol. 9, no. 2, pp. 1109-1112, 1999.

[21] P. Bauer et al., "Minimum quench energies of LHC strands," IEEE Trans. Appl. Supercond., vol. 9, no. 2, pp. 1137-1140, 1999.

[22] A. Devred, "Investigation of the normal zone along a superstabilized superconducting solenoid," J. Appl. Phys., vol. 66, no. 6, Sep. 15, 1989.

[23] M. N. Wilson and R. Wolf, "Calculation of minimum quench energies in Rutherford cables," IEEE Trans. Appl. Sup., vol. 7, no. 2, pp. 950-953, 1997.

[24] L. Bottura, M. Calvi, A. Siemko, "Stability analysis of the LHC cables," Cryogenics, 46, pp. 481-493, 2006.

[25] D. Bocian, B. Dehning, A. Siemko, "Modelling of quench limit for steady state heat deposits in LHC magnets," IEEE Trans. Appl. Sup., vol. 18, no. 2, pp. 112-115, 2008.

[26] G. Willering, "Stability of superconducting Rutherford cables for accelerator magnets," PhD dissertation, Low Temperature Div., Univ. Twente, Twente, 2009.

[27] G. P. Willering, A. Verweij, C. Scheuerlein, A. den Ouden, and H. H. ten Kate, "Difference in stability between edge and center in a rutherford cable," IEEE Trans. Appl. Supercond, vol. 18, no. 2, pp. 1253-1256, 2008.

[28] L. Bottura, "Modeling stability in superconducting cables," Physica C, vol. 310 , pp. $316-326,1998$.
[29] L. Bottura, C. Rosso, M. Breschi, "A General Model for Thermal, Hydraulic and Electric Analysis of Superconducting Cables," Cryogenics, vol. 40, pp. $617-626,2000$.

[30] Thea Software, CryoSoft, France, http://www.htess.com/cryosoft.htm.

[31] A. Akhmetov, L. Bottura, M. Breschi, P. L. Ribani, "A theoretical investigation on current imbalance in flat two-layer superconducting cables," Cryogenics, Vol. 40, n. 9-10, pp. 627 - 635, 2000.

[32] L. Bottura, M. Breschi, A. Musso, "Calculation of interstrand coupling losses in superconducting Rutherford cables with a continuum model," Cryogenics, vol. 96, pp. 44-52, 2018.

[33] P. P. Granieri, R. van Weelderen, "Deduction of Steady-State Cable Quench Limits for Various Electrical Insulation Schemes With Application to LHC and HL-LHC Magnets," IEEE Trans. Appl. Supercond., 24 4802806, 2014.

[34] P. P. Granieri, M. Calvi, D. Bocian, L. Bottura, M. Breschi, A. Siemko, "Stability Analysis of the LHC Cables for Transient Heat Depositions," IEEE Trans. on Appl. Supercond., vol. 18, n. 2, pp. 1257 - 1262, 2008

[35] P. Bauer, "Stability of Superconducting Strand for Accelerator Magnets," $\mathrm{PhD}$. Dissertation, Technische Universität Wien, 1996.

[36] J. Lantz, "Heat Transfer Correlations Between a Heated Surface and Liquid \& Superfluid Helium," Ph. D. Dissertation, LinkopingsUniversitet, 2007.

[37] P. Galassi, Stability maps of the LHC superconducting magnets, Master thesis, University of Bologna, 2013.

[38] M. Breschi, A. Bevilacqua, L. Bottura, P. P. Granieri, "Analysis of BeamInduced Quenches of the LHC Cables with a Multi-Strand Model," IEEE Trans. Appl. Supercond. vol. 25, n. 3, Article Number 4700405, 2015.

[39] M. Breschi, E. Felcini, L. Bottura, "Quench Level of the HL-LHC Nb 33 IR Quadrupoles," IEEE Trans. Appl. Supercond., vol. 27, n. 4, Article Number 7782864, 2017.

[40] T. Winkler, "Helium II Heat Transfer in LHC Magnets," PhD dissertation, Low Temperature Div., University of Twente, Twente, 2017.

[41] L. Bottura, M. Breschi, E. Felcini, A. Lechner, "Stability margin of the LHC Nb-Ti Magnets subjected to beam losses," submitted for publication on Physical Review Accelerators and Beams, 2019.

[42] E. Felcini, "Analysis of the Stability Margin of the High Luminosity LHC Superconducting Cables with a Multi-Strand Model," Master thesis, University of Bologna, 2016. 